\title{
Effectiveness of nutrition interventions and combined nutrition and physical activity interventions in older adults with frailty or prefrailty: a systematic review and meta-analysis
}

\author{
Megan Racey PhD, Muhammad Usman Ali MD MSc, Diana Sherifali RN PhD, \\ Donna Fitzpatrick-Lewis MSW, Ruth Lewis BA, Milos Jovkovic MSc, Leah Gramlich MD, \\ Heather Keller RD PhD, Jayna Holroyd-Leduc MD, Anik Giguère PhD, Ada Tang PhD, \\ Danielle Bouchard PhD, Jeanette Prorok PhD, Perry Kim PhD, Amanda Lorbergs PhD, \\ John Muscedere MD; for the Canadian Frailty Network
}

See related research by Racey and colleagues at www.cmajopen.ca/lookup/doi/10.9778/cmajo.20200222

\section{Abstract}

Background: Although nutrition interventions may reverse frailty, it is unclear which interventions are optimal. We conducted a systematic review and meta-analysis to identify effective nutrition interventions that improve outcomes related to frailty.

Methods: We searched MEDLINE, Embase, the Cochrane Central Register of Controlled Trials and CINAHL (inception to July 2019) for English-language trials of nutrition and combined-approach (nutrition and exercise) interventions (with a control group) involving prefrail or frail adults aged 65 years or more. Outcomes of interest were frailty, mobility, health (body weight, body mass index), physical function (e.g., activities of daily living, muscle strength, appendicular lean mass), use of health care services (e.g., hospital admissions), quality of life, diet quality, mortality, and caregiver and social support. After data extraction, we assessed the risk of bias using the Cochrane Collaboration risk of bias tool for randomized controlled trials, rated the certainty of evidence with the Grading of Recommendation, Assessment, Development and Evaluations (GRADE) approach, and assessed statistical and methodologic heterogeneity. In addition, we conducted subgroup analyses of studies based on the primary intervention of protein supplementation.

Results: We identified 15 studies involving 1825 prefrail or frail older participants: 7 were nutrition trials, 7 were combined-approach trials, and 1 trial had both a nutrition arm and a combined-approach arm. Seven studies had low risk of bias, 2 studies had high risk of bias, and for 6 studies the risk of bias was unclear. Nutrition interventions had small but significant effects on measures of physical function (standardized mean difference [SMD] $0.16,95 \%$ confidence interval $[\mathrm{Cl}] 0.02$ to 0.29 ), mobility (SMD $0.15,95 \% \mathrm{Cl} 0.00$ to 0.30 ) and frailty (SMD $-0.22,95 \% \mathrm{Cl}-0.44$ to -0.01 ) outcomes. Nutrition interventions combined with physical activity also had small but significant effects on physical function (SMD $0.19,95 \% \mathrm{Cl} 0.06$ to 0.32 ), mobility (SMD $0.25,95 \% \mathrm{Cl} 0.02$ to 0.48 ) and frailty (SMD $-0.41,95 \% \mathrm{Cl}-0.68$ to -0.14 ; risk ratio $0.72,95 \% \mathrm{Cl} 0.52$ to 1.00 ) measures. Protein supplementation (5 studies) had small but significant effects on physical function measures (SMD $0.16,95 \% \mathrm{Cl} 0.01$ to 0.31 ) and mobility measures (SMD $0.20,95 \% \mathrm{Cl}$ 0.02 to 0.39 ), with moderate certainty of evidence.

Interpretation: There is moderate evidence that nutrition (including protein supplementation) and combined interventions are beneficial for prefrail or frail older adults. Trials with clear definitions of frailty and outcomes that reflect frailty identification and diagnosis are needed. PROSPERO registration: CRD42020144819

F railty is a leading contributor to functional decline and early death in older adults. ${ }^{1}$ More than 1.5 million Canadians are estimated to be diagnosed as medically frail, and this number is expected to rise to more than 2 million in the next 10 years. $^{2}$ Frailty is a syndrome resulting from multiple factors and impairments that can reduce a person's functional ability. It is characterized by reductions in physiologic reserve and a reduced ability to respond to stress. ${ }^{3,4}$ Older adults with frailty are at increased risk for
Competing interests: Jeanette Prorok, Perry Kim, Amanda Lorbergs and John Muscedere are salaried employees of the Canadian Frailty Network. No other competing interests were declared.

This article has been peer reviewed.

Correspondence to: Diana Sherifali, dsherif@mcmaster.ca; Megan Racey, raceym@mcmaster.ca

CMAJ Open 2021. DOI:10.9778/cmajo.20200248 
adverse outcomes such as falls, decline in mobility, hospital admission and death. ${ }^{5,6}$ Proper identification and measurement of frailty is necessary to optimize care and treatment. ${ }^{2}$

Frailty is not a natural outcome of aging. Many adults reach advanced ages without developing frailty, ${ }^{7}$ and progression can be slowed or reversed through nutrition interventions. ${ }^{1,8}$ Since frailty is a multicomponent condition that includes physical factors such as reduced handgrip strength and gait speed, it is important to consider the enhanced impact that adequate nutrition could have on the benefits of physical activity in a frail population. Based on the mixed results from previous reviews looking at both nutrition interventions alone and physical activity interventions with nutrition supplementation, ${ }^{9-11}$ it is still unclear what the best interventions are to support older adults with frailty, especially across a range of outcomes and in a population that has been clearly identified as frail.

We conducted a systematic review and meta-analysis focused on nutrition interventions and nutrition interventions combined with physical activity (combined approach) in an identified prefrail or frail population to assess the effectiveness of the interventions in improving outcomes including frailty, mobility, health (body weight, body mass index), physical function (e.g., activities of daily living, muscle strength, appendicular lean mass), use of health care services (e.g., hospital admissions), quality of life, diet quality, mortality, and caregiver and social support. The results of this review, along with the results of a methodologically similar review focused on physical activity interventions, ${ }^{12}$ will provide the scientific evidence for the clinical practice guidelines of the Canadian Frailty Network.

\section{Methods}

This systematic review and meta-analysis followed the Preferred Reporting Items for Systematic Reviews and Metaanalyses (PRISMA) guidelines. ${ }^{13}$ The protocol was registered in the International Prospective Register of Systematic Reviews (PROSPERO-CRD42020144556). There were minor variations from the published protocol. These included application of the English-only language restriction on screening rather than as part of the search, and reporting on only the outcomes listed in the protocol that were ranked critical based on the voting of a guideline panel committee. Our methods followed the Cochrane Handbook for Systematic Reviews of Interventions. ${ }^{14}$

\section{Key question}

What is the effectiveness of nutrition interventions in older adults (aged $\geq 65 \mathrm{yr}$ ) with frailty or prefrailty on clinical outcomes, outcomes that are important to the patient and outcomes related to use of health care services?

\section{Search strategy}

We developed the search terms, databases and strategy in consultation with a research librarian; they were informed by previous systematic reviews ${ }^{9-11}$ (Appendix 1, available at www.
cmajopen.ca/content/9/3/E744/suppl/DC1). We searched MEDLINE (1948 to July 2019), Embase (1974 to July 2019), the Cochrane Central Register of Controlled Trials (2019, Issue 6) and CINAHL (1937 to July 2019), and manually searched reference lists of relevant reviews and included studies for citations not captured in our search. Results from the search were deduplicated, and citations were uploaded to a secure Internet-based platform for screening (DistillerSR, Evidence Partners).

\section{Eligibility criteria}

The PICO (population, intervention, comparator, outcomes) inclusion and exclusion criteria are provided in Box 1.

\section{Box 1: Population, intervention, comparator, outcomes (PICO) eligibility criteria}

\section{Population}

- Adults aged $\geq 65 \mathrm{yr}$ who were selected for and clearly identified as prefrail or frail with any frailty assessment tool, assessment of frailty or other explicit author-established criteria (including studies with subanalyses involving a portion of prefrail or frail participants)

- To make this review relevant to the general frail population, studies that targeted clinical populations, such as patients with obesity or cancer, were excluded

Intervention

- Nutrition interventions involved a nutrition component such as food supplementation, meal programs and education

- Combined-approach interventions must have included both nutrition and physical activity components in the same intervention; physical activity was defined as any bodily movement by skeletal muscles that results in energy expenditure above resting levels, and included various patterns, frequencies, durations, intensities and types

- Studies that included other intervention components, such as memory tasks, were excluded

Comparator

- True control group defined as usual care, routine care or minimal contact that did not include any intervention or intervention group components

- Any head-to-head interventions were excluded

Outcomes

- Frailty (measured with a valid tool)

- Mobility (e.g., gait speed, Timed Up and Go test, Sit-to-Stand test, balance test, Short Physical Performance Battery)

- Health (body weight, body mass index)

- Physical function (e.g., activities of daily living, muscle strength [handgrip and nonhandgrip], appendicular lean mass)

- Use of health care services (e.g., hospital admission, emergency department visits)

- Quality of life (measured with a standardized tool)

- Diet quality (energy in kilocalories)

- Mortality

- Caregiver and social support (measured with the Zarit Burden Interview, Caregiver Burden Scale, Revised Scale for Caregiving Self-Efficacy and Caregiver Reaction Assessment) 
We included peer-reviewed studies of interventions with a control group (randomization was not required) available in English. There was no exclusion based on intervention or participant setting.

Outcomes of interest were selected by an interdisciplinary steering committee (L.G., H.K., J.H.-L., A.G., A.T., D.B., J.M.) through a voting process that involved gathering a comprehensive list of outcomes from clinical and research expertise as well as existing relevant systematic reviews. The committee identified any missing outcomes and then anonymously ranked the outcomes on a scale from 1-9 (1-3: not important; 4-6: important; 7-9: critical). Authors not involved in the ranking process (D.S., D.F-L., M.R.) averaged the scores for each outcome and provided the list of outcomes to the committee for final discussion and agreement.

\section{Study selection, data extraction and quality assessment}

A series of team members were involved in the screening process (title and abstract, and full text), which resulted in different combinations of reviewers and team members. At all levels of screening, all articles required a minimum of 2 reviews. Multiple publications for the same primary intervention were merged; all data from all publications were extracted for relevant outcomes, but we only describe the study and its characteristics once in our review.

We developed, piloted and deployed standardized forms for data extraction. Two team members independently completed full data extraction (intention-to-treat data) and assessment of study risk of bias using the Cochrane Collaboration risk of bias tool $^{15}$ for randomized controlled trials (RCTs). If interventions had multiple treatment arms, only the interventions that met our inclusion criteria were extracted. All extraction was verified independently by a statistician (M.U.A.). Conflicts were resolved by the lead researcher of this review (M.R.).

Data on harms or adverse events were extracted narratively.

\section{Certainty of evidence}

We independently evaluated the certainty of the body of evidence using the Grading of Recommendation, Assessment, Development and Evaluations (GRADE) approach ${ }^{16}$ with GRADEpro software. With this method, the certainty of a body of evidence is rated as high, moderate, low or very low based on assessment of 5 conditions: methodologic quality; consistency across effect estimates and statistical heterogeneity; directness of the body of evidence to the populations, interventions, comparators or outcomes of interest; precision of results; and indications of reporting bias.

\section{Statistical analysis}

All data analysis were planned a priori (Appendix 2, available at www.cmajopen.ca/content/9/3/E744/suppl/DC1). We used a meta-analysis to combine the results across studies by intervention category and outcome using the published data from included studies (full methods in Appendix 3, available at www.cmajopen.ca/content/9/3/E744/suppl/DC1). For continuous outcomes, we used the change from baseline to immediately after the intervention (mean change score, standard deviation [SD] of mean change score) for both intervention and control groups to generate the summary measures of effect in the form of standardized mean difference (SMD). ${ }^{17}$ We used the SMD as a summary statistic because many studies in this systematic review assessed the same outcome measured in a variety of ways (e.g., mobility measured as Stair Climb Test, balance test, gait speed, chair rise repetition, Sitto-Stand test, Short Physical Performance Battery, Timed Up and Go test). In this situation, it was necessary to standardize the results of the studies before they could be compared across studies or combined in a quantitative synthesis. The SMD-based effect sizes represent the magnitude of intervention effect relative to the variability observed within a particular study. Therefore, the studies for which the difference in mean change score was the same as the proportion of SD of mean change score will have the same SMD, regardless of the actual scale or unit of measurement used to access the outcome measures. ${ }^{18,19}$ The SMD is interpreted based on its magnitude according to recommended Cohen $d$ thresholds $(\sim 0.2=$ small effect, $\sim 0.5=$ medium effect, $\sim 0.8=$ large effect). ${ }^{20}$ For studies in which measure of variance was reported as confidence intervals (CIs), standard error or $p$ values, we used Cochrane-recommended methods to convert these data to SD. ${ }^{18}$

We used a multilevel meta-analytical approach (where applicable) to account for statistical dependence, that is, dependency in effect sizes introduced by comparison of multiple intervention arms within a study to a common control group, or by multiple outcome measures or suboutcome measures of a primary outcome of interest within a study (such as the Short Physical Performance Battery reported as gait speed, balance test and Chair Stand Test separately). In such cases, we nested the correlated measures or effect sizes within studies first, by introducing a random effect to our grouping variables such as studies, outcome measures and intervention arms. This grouping variable, also known as random intercept, told our model to assume different values (intercepts) for each grouping level. Specifically, we used 2 grouping variables: that on level 2, and that on level 3. We assumed that these grouping variables are nested, in the sense that several effect sizes on level 2 together make up a larger cluster on level 3. For pooling of performance measures, we adjusted the direction of effect to ensure consistency of desirable outcome responses.

For dichotomous outcomes, we used the number of events after the intervention to generate the summary measures of effect in the form of risk ratio (RR) using DerSimonian and Laird random-effects models with the Mantel-Haenszel method. We used the Cochran Q $(\alpha=0.05)$ to detect statistical heterogeneity and the $I^{2}$ statistic to quantify the magnitude of statistical heterogeneity between studies, where an $I^{2}$ value of $30 \%-60 \%$ represents moderate heterogeneity and a value of $60 \%-90 \%$ represents substantial heterogeneity across studies.

We estimated the statistical heterogeneity $I^{2}$ statistic in the context of the multilevel meta-analytical approach. ${ }^{19,21} \mathrm{We}$ 
estimated $I^{2}$-level 2 to account for the amount of withincluster heterogeneity (i.e., across effect sizes or multiple arms of same study), $I^{2}$-level 3 to account for between-cluster heterogeneity (i.e., effect sizes across studies or subgroups of interest) and $I^{2}$-total to represent the heterogeneity not attributable to sample error (sum of values at levels 2 and 3). We added $I^{2}$-total to all forest plots for overall pooled effect estimates and summary effect size within subgroups.

We assessed publication bias using funnel plots when there were at least 10 studies in the meta-analysis. ${ }^{22}$

In addition, we conducted subgroup analyses of studies based on the primary intervention of protein supplementation.

All analyses were performed with $\mathrm{R}$ software (metaphor and dmetar packages) (R Foundation for Statistical Computing).

\section{Ethics approval}

As this study was solely literature based, it was not eligible for institutional ethics approval, and none was sought.

\section{Results}

From 3162 citations, we assessed 123 full-text articles for eligibility, and included 15 studies described in 26 publications (Figure 1). One of the included studies consisted of both a nutrition-only treatment arm and a combined-approach treatment $\mathrm{arm}^{23}$ and was therefore considered in the meta-analysis and qualitative description of both intervention categories. Of the remaining citations, 7 were nutrition $\mathrm{RCTS}^{8,22,24-28}$ and 7 were combined-approach RCTs or clinical controlled trials. ${ }^{29-35} \mathrm{We}$ meta-analyzed all 15 studies and their outcomes based on their intervention category. The studies were published from 2000 to 2019. A total of 1825 frail participants aged 65 years or more (mean 70.0-83.1 yr) were included in this review; the proportion of women ranged from $51 \%$ to $100 \%$. All included studies had fewer than 250 participants (median 89, quartiles 71, 89, 166, 246).

Characteristics of the included studies are presented in Table 1, and further demographic data from studies can be found in Appendix 4 (available at www.cmajopen.ca/content/ 9/3/E744/supp1/DC1). Most interventions were conducted in Europe and Asia with community-dwelling participants and lasted between 3 and 6 months. The most common tool to measure frailty status was Fried's frailty phenotype (also known as the Cardiovascular Health Study criteria $\left.{ }^{47}\right)(n=10)$.

In the nutrition studies, interventions included any or all of the following: nutrition supplementation $(n=4)$, fortified or enhanced foods $(n=7)$, and nutrition or dietitian counselling $(n=1)$. Combined-approach studies also used these 3 nutrition interventions, with 5 studies adding a resistance or strength training component. The physical activity occurred 1-2 times per week and lasted 30 minutes to more than 1 hour.

Adverse effects or harms related to the intervention were reported in 5 studies and included nausea, diarrhea, dyspepsia and acute illness with nutrition interventions, and back pain or other pain related to exercise and heavy study burden with combined-approach interventions.

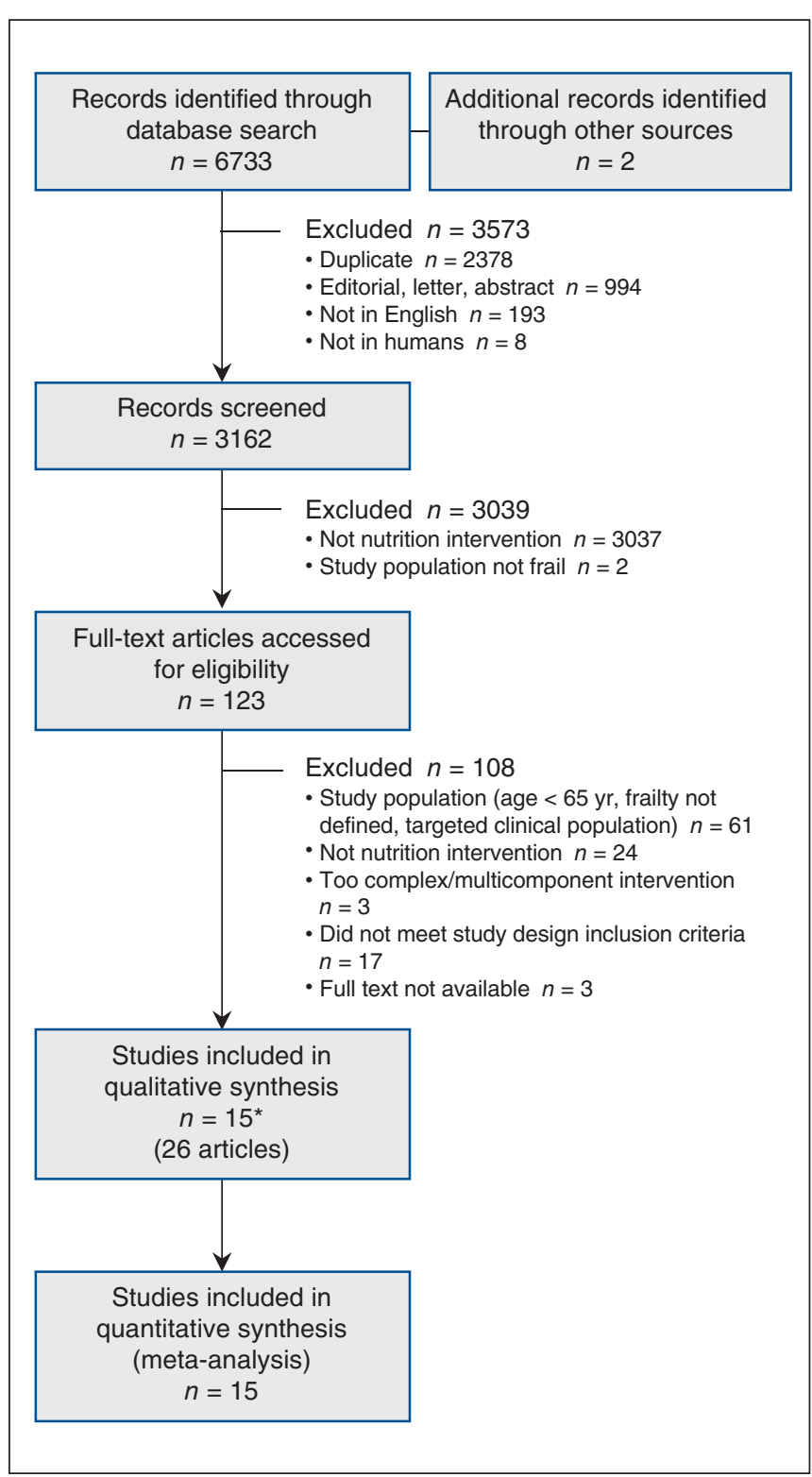

Figure 1: Flow diagram showing study selection. ${ }^{*}$ One citation with 3 articles had both a nutrition-only treatment arm and a combinedapproach treatment arm.

\section{Risk of bias and quality}

The Cochrane Collaboration risk of bias tool showed that 7 studies had low risk of bias, $8,22,26-28,30,332$ studies had high risk of bias, ${ }^{29,35}$ and for 6 studies the risk of bias was unclear, mostly owing to unclear allocation and blinding procedures $^{23-25,31,32,34}$ (Appendix 5, available at www.cmajopen.ca/ content/9/3/E744/suppl/DC1).

The certainty of evidence ranged from very low to moderate but was moderate for most outcomes owing to downgrading for risk of bias or imprecision (Appendix 6, available at www.cmajopen.ca/content/9/3/E744/suppl/DC1).

We did not observe any significant asymmetry across funnel plots for publication bias (Appendix 7, available at www. cmajopen.ca/content/9/3/E744/suppl/DC1). 


\begin{tabular}{|c|c|c|c|c|c|c|c|}
\hline $\begin{array}{l}\text { Study, year; } \\
\text { country }\end{array}$ & $\begin{array}{c}\text { No. of } \\
\text { participants } \\
\text { (F/M, \%) }\end{array}$ & $\begin{array}{l}\text { Age, mean } \\
\pm S D,{ }^{*} \mathrm{yr}\end{array}$ & $\begin{array}{l}\text { Assessment } \\
\text { tool or measure }\end{array}$ & $\begin{array}{c}\text { Design; } \\
\text { duration† }\end{array}$ & Intervention & Control & Outcomes \\
\hline \multicolumn{8}{|c|}{ Nutrition interventions } \\
\hline $\begin{array}{l}\text { Wu et al., }{ }^{24} \\
\text { 2018; Taiwan }\end{array}$ & $40(56 / 44 \ddagger)$ & $\begin{array}{l}\text { O: } 74.0 \\
\text { I: } 73.5 \pm 2.4 \\
75.0 \pm 2.4,72.8 \\
\pm 1.6 \\
C: 75.9 \pm 1.7\end{array}$ & $\begin{array}{l}\text { Fried's frailty } \\
\text { phenotype§ }\end{array}$ & $\begin{array}{l}\text { RCT; } \\
3 \text { mo }\end{array}$ & $\begin{array}{l}\text { Oral nutrition } \\
\text { supplements, } \\
\text { fortified/enhanced } \\
\text { foods, nutrition/ } \\
\text { dietitian } \\
\text { counselling }\end{array}$ & $\begin{array}{l}\text { General } \\
\text { nutrition } \\
\text { information }\end{array}$ & $\begin{array}{l}\text { Health (and death), } \\
\text { physical function } \\
\text { (and quality of life), } \\
\text { mobility, diet quality }\end{array}$ \\
\hline $\begin{array}{l}\text { Niccoli et al., }{ }^{25} \\
\text { 2017; Canada }\end{array}$ & $53(68 / 32 \ddagger)$ & $\begin{array}{l}\mathrm{O}: 81.3 \pm 1.0 \\
\mathrm{I}: 81.77 \pm 1.68 \\
\mathrm{C}: 80.33 \pm 1.57\end{array}$ & $\begin{array}{l}\text { Fried's frailty } \\
\text { phenotype§ }\end{array}$ & $\begin{array}{l}\text { RCT; } \\
\sim 3-4 \text { wk }\end{array}$ & $\begin{array}{l}\text { Fortified/enhanced } \\
\text { foods }\end{array}$ & $\begin{array}{l}\text { Control food } \\
\text { without } \\
\text { supplements }\end{array}$ & $\begin{array}{l}\text { Physical function } \\
\text { (and quality of life), } \\
\text { frailty, mobility }\end{array}$ \\
\hline $\begin{array}{l}\text { Park et al., }{ }^{26} \\
\text { 2018; Korea }\end{array}$ & $120(65 / 35)$ & $\begin{array}{l}\mathrm{I}: 77.30 \pm 3.67 \\
76.80 \pm 3.70 \\
\mathrm{C}: 76.83 \pm 3.86\end{array}$ & $\begin{array}{l}\text { Cardiovascular } \\
\text { Health Study } \\
\text { criteria§ }\end{array}$ & $\begin{array}{l}\text { RCT; } \\
12 \text { wk }\end{array}$ & $\begin{array}{l}\text { Fortified/enhanced } \\
\text { foods }\end{array}$ & $\begin{array}{l}\text { Placebo } \\
\text { powder }\end{array}$ & $\begin{array}{l}\text { Physical function } \\
\text { (and quality of life), } \\
\text { frailty, mobility, diet } \\
\text { quality }\end{array}$ \\
\hline $\begin{array}{l}\text { Ng et al. }{ }^{8,36,37} \\
2015 ; \\
\text { Singapore }\end{array}$ & $246(61 / 39)$ & $\begin{array}{l}\text { O: } 70.0 \pm 4.7 \\
\text { I: } 69.7 \pm 4.23 \\
\text { C: } 70.1 \pm 5.02\end{array}$ & $\begin{array}{l}\text { Cardiovascular } \\
\text { Health Study } \\
\text { criteria§ }\end{array}$ & $\begin{array}{l}\mathrm{RCT} \\
6 \mathrm{mo}\end{array}$ & $\begin{array}{l}\text { Oral nutrition } \\
\text { supplements, } \\
\text { fortified/enhanced } \\
\text { foods }\end{array}$ & $\begin{array}{l}\text { Placebo } \\
\text { supplement }\end{array}$ & $\begin{array}{l}\text { Health (and death), } \\
\text { physical function } \\
\text { (and quality of life), } \\
\text { use of health care } \\
\text { services, frailty, } \\
\text { mobility }\end{array}$ \\
\hline $\begin{array}{l}\text { Kim et al.. }{ }^{27} \\
\text { 2013; South } \\
\text { Korea }\end{array}$ & $87(79 / 21)$ & $\begin{array}{l}\mathrm{I}: 78.9 \pm 5.5 \\
\mathrm{C}: 78.4 \pm 6.0\end{array}$ & $\begin{array}{l}\text { Slow gait } \\
\text { speed, MNA }\end{array}$ & $\begin{array}{l}\text { RCT; } \\
12 \text { wk }\end{array}$ & $\begin{array}{l}\text { Oral nutrition } \\
\text { supplements, } \\
\text { fortified/enhanced } \\
\text { foods }\end{array}$ & $\begin{array}{l}\text { No contact or } \\
\text { care }\end{array}$ & $\begin{array}{l}\text { Health (and death), } \\
\text { physical function } \\
\text { (and quality of life), } \\
\text { frailty, mobility, diet } \\
\text { quality }\end{array}$ \\
\hline $\begin{array}{l}\text { Tieland } \\
\text { et al., } 28,38 \\
\text { 2012; the } \\
\text { Netherlands }\end{array}$ & $65(55 / 45)$ & $\begin{array}{l}\mathrm{O}: 83.1 \pm 5.1 \\
\mathrm{I}: 78.0 \pm 1 \\
\mathrm{C}: 81.0 \pm 1\end{array}$ & $\begin{array}{l}\text { Fried's frailty } \\
\text { phenotype }\end{array}$ & $\begin{array}{l}\mathrm{RCT} \\
24 \text { wk }\end{array}$ & $\begin{array}{l}\text { Fortified/enhanced } \\
\text { foods }\end{array}$ & $\begin{array}{l}\text { Placebo } \\
\text { supplement }\end{array}$ & $\begin{array}{l}\text { Health (and death), } \\
\text { physical function } \\
\text { (and quality of life), } \\
\text { mobility, diet quality }\end{array}$ \\
\hline $\begin{array}{l}\text { Latham } \\
\text { et al., }{ }^{22} 2003 ; \\
\text { New Zealand }\end{array}$ & $243(53 / 47)$ & $\begin{array}{l}\text { O: } 79.1 \pm 6.9 \\
\text { I: } 79.0(95 \% \mathrm{Cl} \\
77 \text { to } 80) \\
\text { C: } 80.0(95 \% \mathrm{Cl} \\
78 \text { to } 81)\end{array}$ & $\begin{array}{l}\text { Clinical } \\
\text { measures of } \\
\text { frailty§ }\end{array}$ & $\begin{array}{l}\mathrm{RCT} \\
3 \mathrm{mo}\end{array}$ & $\begin{array}{l}\text { Oral nutrition } \\
\text { supplements }\end{array}$ & $\begin{array}{l}\text { Placebo } \\
\text { supplement }\end{array}$ & $\begin{array}{l}\text { Physical function } \\
\text { (and quality of life), } \\
\text { mobility }\end{array}$ \\
\hline $\begin{array}{l}\text { de Jong } \\
\text { et al., 23,39,40 } \\
2000 ; \uparrow \text { the } \\
\text { Netherlands }\end{array}$ & $217(70 / 30)$ & $\begin{array}{l}\text { O: } 79.0 \\
\text { I: } 79.6 \pm 4.8 \\
\text { C: } 79.3 \pm 6.6\end{array}$ & $\begin{array}{l}\text { Required health } \\
\text { care service } \\
\text { (e.g., home } \\
\text { care, meal } \\
\text { delivery) }\end{array}$ & $\begin{array}{l}\text { RCT } \\
17 \text { wk }\end{array}$ & $\begin{array}{l}\text { Fortified/enhanced } \\
\text { foods }\end{array}$ & $\begin{array}{l}\text { Control food } \\
\text { without } \\
\text { supplements }\end{array}$ & $\begin{array}{l}\text { Physical function } \\
\text { (and quality of life), } \\
\text { mobility, diet quality }\end{array}$ \\
\hline \multicolumn{8}{|c|}{ Combined-approach interventions } \\
\hline $\begin{array}{l}\text { Kang et al., }{ }^{29} \\
\text { 2019; China }\end{array}$ & $115(62 / 38)$ & $\begin{array}{l}\text { O: } 77.3 \\
\text { I: } 76.79 \pm 7.11 \\
78.04 \pm 6.82\end{array}$ & $\begin{array}{l}\text { Fried's frailty } \\
\text { phenotype§ }\end{array}$ & $\begin{array}{l}\text { CCT; } \\
12 \text { wk }\end{array}$ & $\begin{array}{l}\text { Fortified/enhanced } \\
\text { foods, muscle } \\
\text { strengthening }\end{array}$ & $\begin{array}{l}\text { Information } \\
\text { about diet to } \\
\text { maintain } \\
\text { current } \\
\text { weight, usual } \\
\text { daily exercise } \\
\text { program }\end{array}$ & $\begin{array}{l}\text { Health (and death), } \\
\text { physical function } \\
\text { (and quality of life), } \\
\text { mobility }\end{array}$ \\
\hline $\begin{array}{l}\text { Serra-Prat } \\
\text { et al., }{ }^{30} 2017 \text {; } \\
\text { Spain }\end{array}$ & $172(56 / 44)$ & $\begin{array}{l}\text { O: } 78.3 \\
\text { I: } 77.9 \pm 5.0 \\
\text { C: } 78.8 \pm 4.9\end{array}$ & $\begin{array}{l}\text { Fried's frailty } \\
\text { phenotype }\end{array}$ & $\begin{array}{l}\text { RCT; } \\
12 \text { mo }\end{array}$ & $\begin{array}{l}\text { Nutrition/dietitian } \\
\text { counselling, mixed } \\
\text { physical activity } \\
\text { (muscle } \\
\text { strengthening/ } \\
\text { aerobic/exercises) }\end{array}$ & Usual care & $\begin{array}{l}\text { Frailty, health (and } \\
\text { death), physical } \\
\text { function (and quality } \\
\text { of life), mobility }\end{array}$ \\
\hline $\begin{array}{l}\text { Luger } \\
\text { et al.,31,41-44 } \\
\text { 2016; Austria }\end{array}$ & $80(84 / 16)$ & $\begin{array}{l}\text { O: } 82.8 \pm 8.0 \\
\mathrm{I}: 83.0 \pm 8.1 \\
\mathrm{C}: 82.5 \pm 8.0\end{array}$ & SHARE-FI§ & $\begin{array}{l}\text { RCT; } \\
12 \text { wk }\end{array}$ & $\begin{array}{l}\text { Nutrition/dietitian } \\
\text { counselling, } \\
\text { muscle } \\
\text { strengthening }\end{array}$ & $\begin{array}{l}\text { Visited } 2 \times / \text { wk } \\
\text { by "buddies" }\end{array}$ & $\begin{array}{l}\text { Frailty, physical } \\
\text { function (and quality } \\
\text { of life), mobility }\end{array}$ \\
\hline
\end{tabular}




\begin{tabular}{|c|c|c|c|c|c|c|c|}
\hline $\begin{array}{l}\text { Study, year; } \\
\text { country }\end{array}$ & $\begin{array}{c}\text { No. of } \\
\text { participants } \\
\text { (F/M, \%) }\end{array}$ & $\begin{array}{l}\text { Age, mean } \\
\pm \mathrm{SD},{ }^{*} \mathrm{yr}\end{array}$ & $\begin{array}{l}\text { Assessment } \\
\text { tool or measure }\end{array}$ & $\begin{array}{l}\text { Design; } \\
\text { duration† }\end{array}$ & Intervention & Control & Outcomes \\
\hline $\begin{array}{l}\text { Kwon et al., }{ }^{32} \\
2015 \text {; Japan }\end{array}$ & $89(100 / 0)$ & $\begin{array}{l}\text { O: } 76.8 \\
\text { I: } 76.5 \pm 3.8 \\
77.0 \pm 4.2 \\
C: 76.9 \pm 3.9\end{array}$ & $\begin{array}{l}\text { Fried's frailty } \\
\text { phenotype§ }\end{array}$ & $\begin{array}{l}\mathrm{RCT} \\
3 \mathrm{mo}\end{array}$ & $\begin{array}{l}\text { Nutrition/dietitian } \\
\text { counselling, } \\
\text { muscle } \\
\text { strengthening }\end{array}$ & $\begin{array}{l}\text { General } \\
\text { health } \\
\text { education } \\
\text { session } \\
1 \times / \text { mo }\end{array}$ & $\begin{array}{l}\text { Frailty, physical } \\
\text { function (and quality } \\
\text { of life), mobility }\end{array}$ \\
\hline $\begin{array}{l}\text { Tieland } \\
\text { et al.,33,45,46 } \\
\text { 2012; the } \\
\text { Netherlands }\end{array}$ & $62(66 / 34)$ & $\begin{array}{l}\text { I: } 78 \pm 9 \\
\text { C: } 79 \pm 6\end{array}$ & $\begin{array}{l}\text { Fried's frailty } \\
\text { phenotype }\end{array}$ & $\begin{array}{l}\text { RCT; } \\
24 \text { wk }\end{array}$ & $\begin{array}{l}\text { Fortified/enhanced } \\
\text { foods, muscle } \\
\text { strengthening }\end{array}$ & $\begin{array}{l}\text { Exercise } \\
\text { training } 2 \times / \\
\text { wk, placebo } \\
\text { supplement } \\
2 \times / d\end{array}$ & $\begin{array}{l}\text { Health (and death), } \\
\text { physical function } \\
\text { (and quality of life), } \\
\text { mobility, diet quality }\end{array}$ \\
\hline $\begin{array}{l}\text { Yamada } \\
\text { et al., }{ }^{35} 2012 \text {; } \\
\text { Japan }\end{array}$ & $77(51 / 49 \ddagger)$ & $\begin{array}{l}\mathrm{I}: 74.4 \pm 7.3 \\
\mathrm{C}: 75.6 \pm 6.0\end{array}$ & $\begin{array}{l}\text { Frailty status as } \\
\text { certified by } \\
\text { long-term care } \\
\text { insurance } \\
\text { service }\end{array}$ & $\begin{array}{l}\text { CCT; } \\
3 \mathrm{mo}\end{array}$ & $\begin{array}{l}\text { Oral nutrition } \\
\text { supplements, } \\
\text { fortified/enhanced } \\
\text { foods, muscle } \\
\text { strengthening }\end{array}$ & $\begin{array}{l}\text { Both groups } \\
\text { received } \\
\text { exercise } \\
\text { training }\end{array}$ & $\begin{array}{l}\text { Physical outcomes } \\
\text { (and quality of life), } \\
\text { mobility }\end{array}$ \\
\hline $\begin{array}{l}\text { de Jong } \\
\text { et al., }{ }^{23,39,40} \\
2000 ;^{* *} \text { the } \\
\text { Netherlands }\end{array}$ & $217(70 / 30)$ & $\begin{array}{l}\text { O: } 79.0 \\
\text { I: } 79.2 \pm 6.1 \\
\text { C: } 79.3 \pm 6.6\end{array}$ & $\begin{array}{l}\text { Required health } \\
\text { care service } \\
\text { (e.g., home } \\
\text { care, meal } \\
\text { delivery) }\end{array}$ & $\begin{array}{l}\mathrm{RCT} \\
17 \text { wk }\end{array}$ & $\begin{array}{l}\text { Fortified/enhanced } \\
\text { foods, mixed } \\
\text { physical activity } \\
\text { (muscle } \\
\text { strengthening/ } \\
\text { aerobic/exercises) }\end{array}$ & $\begin{array}{l}\text { Control food, } \\
\text { social } \\
\text { program once } \\
\text { every } 2 \mathrm{wk} \\
\text { for } 90 \mathrm{~min}\end{array}$ & $\begin{array}{l}\text { Physical function } \\
\text { (and quality of life), } \\
\text { mobility, diet quality }\end{array}$ \\
\hline $\begin{array}{l}\text { Nykänen } \\
\text { et al., }{ }^{34} 2012 ; \\
\text { Finland }\end{array}$ & $159(79 / 21)$ & $\begin{array}{l}\text { O: } 83.1 \pm 5.1 \\
\mathrm{I}: 83.2 \pm 5.2 \\
\mathrm{C}: 82.9 \pm 5.0\end{array}$ & $\begin{array}{l}\text { Cardiovascular } \\
\text { Health Study } \\
\text { criteria§ }\end{array}$ & CCT; 1 yr & $\begin{array}{l}\text { Nutrition/dietitian } \\
\text { counselling, mixed } \\
\text { physical activity } \\
\text { (muscle } \\
\text { strengthening/ } \\
\text { aerobic/exercises) }\end{array}$ & Usual care & Frailty, diet quality \\
\hline \multicolumn{8}{|c|}{ 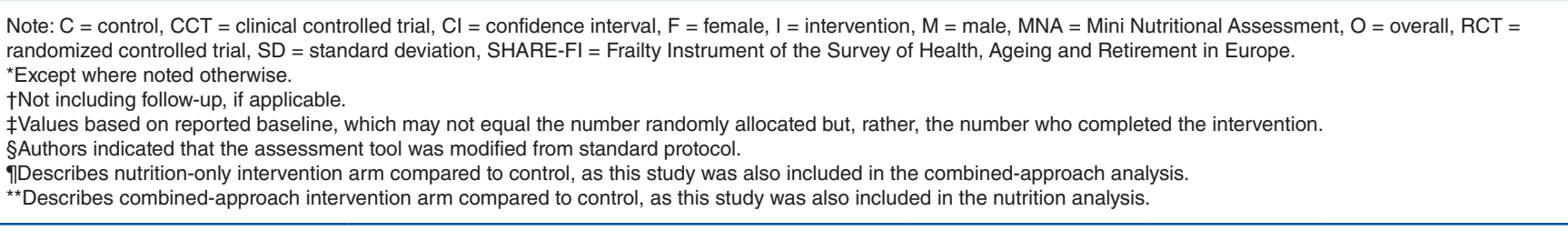 } \\
\hline
\end{tabular}

\section{Benefits of treatment}

The meta-analysis for all outcomes included an examination of the impact of all nutrition interventions together, all combined-approach interventions together and a subgroup of protein supplementation interventions (Table 2).

\section{Nutrition interventions}

Nutrition interventions supplemented protein, ${ }^{24-26,28}$ other multinutrients and multivitamins, ${ }^{8,24,27}$ vitamin $\mathrm{D},{ }^{22}$ and fruit and dairy products ${ }^{23}$ (Appendix 4, Supplemental Table S1). Overall, nutrition RCTs had small but significant effects on physical, mobility and frailty measures, with moderate certainty of evidence (Table 2; Appendix 6, Supplemental Table S4). There were no significant effects on measures of health, diet quality or quality of life (Table 2; Appendix 8, Supplemental Figures S7-S9, available at www.cmajopen.ca/ content/9/3/E744/suppl/DC1).

The 7 RCTs $^{8,22,24-28}$ (373 intervention and 321 control participants) had a small but significant pooled effect estimate for physical measures (SMD 0.16, 95\% CI 0.02 to 0.29) (Figure 2A). A similar effect was observed for mobility measures (SMD 0.15, 95\% CI 0.00 to 0.30) (Figure 2B).

Frailty outcomes were reported in $3 \mathrm{RCTs}^{8,24,26}$ from 155 intervention and 100 control participants. These studies had a small but significant pooled effect estimate for frailty (SMD $-0.22,95 \% \mathrm{CI}-0.44$ to -0.01 ) (Figure 2C).

\section{Protein supplementation interventions}

We identified 5 studies for subgroup analysis based on the primary intervention of protein supplementation. ${ }^{24-28}$ These RCTs had 195 intervention and 149 control participants and ranged in duration from 4 to 24 weeks. There were small but significant effects on physical measures (SMD 0.16, 95\% CI 0.01 to 0.31 ) (Figure $3 \mathrm{~A}$ ) and mobility measures (SMD 0.20, $95 \%$ CI 0.02 to 0.39 ) (Figure 3B), with moderate certainty of evidence (Table 2; Appendix 6, Supplemental Table S5). There were no significant effects on measures of health, frailty or diet quality (Appendix 8, Supplemental Figures S10-S12). 
Table 2: Effect estimates for outcomes and certainty of evidence, for all interventions combined and by intervention category

\begin{tabular}{|c|c|c|c|c|}
\hline Category; outcome* $\dagger$ & $\begin{array}{l}\text { No. of } \\
\text { studies }\end{array}$ & $\begin{array}{c}\text { No. of } \\
\text { participants }\end{array}$ & SMD $\ddagger(95 \% \mathrm{CI}) \S$ & GRADE rating \\
\hline \multicolumn{5}{|l|}{ Nutrition interventions } \\
\hline Physical function & 7 & 694 & $0.16(0.02$ to 0.29$)$ & Moderate; downgraded for risk of bias \\
\hline Mobility & 7 & 694 & $0.15(0.001$ to 0.30$)$ & Moderate; downgraded for risk of bias \\
\hline Health & 4 & 284 & $-0.18(-0.51$ to 0.16$)$ & $\begin{array}{l}\text { Low; downgraded for risk of bias and } \\
\text { imprecision }\end{array}$ \\
\hline Frailty & 3 & 255 & $-0.22(-0.44$ to -0.01$)$ & Moderate; downgraded for risk of bias \\
\hline Diet quality & 5 & 383 & $0.10(-0.47$ to 0.67$)$ & $\begin{array}{l}\text { Very low; downgraded for risk of bias, } \\
\text { inconsistency and imprecision }\end{array}$ \\
\hline Quality of life & 1 & 243 & $-0.12(-1.39$ to 1.15$)$ & Moderate; downgraded for imprecision \\
\hline \multicolumn{5}{|c|}{ Protein supplementation interventions } \\
\hline Physical function & 5 & 344 & $0.16(0.01$ to 0.31$)$ & Moderate; downgraded for risk of bias \\
\hline Mobility & 5 & 344 & $0.20(0.02$ to 0.39$)$ & Moderate; downgraded for risk of bias \\
\hline Health & 3 & 177 & $-0.12(-0.58$ to 0.34$)$ & $\begin{array}{l}\text { Low; downgraded for risk of bias and } \\
\text { imprecision }\end{array}$ \\
\hline Frailty & 2 & 148 & $-0.18(-0.45$ to 0.09$)$ & $\begin{array}{l}\text { Low; downgraded for risk of bias and } \\
\text { imprecision }\end{array}$ \\
\hline Diet quality & 4 & 297 & $-0.01(-0.69$ to 0.67$)$ & $\begin{array}{l}\text { Very low; downgraded for risk of bias, } \\
\text { inconsistency and imprecision }\end{array}$ \\
\hline \multicolumn{5}{|c|}{ Combined-approach (nutrition and physical activity) interventions } \\
\hline Physical function & 6 & 514 & 0.19 (0.06 to 0.32$)$ & Moderate; downgraded for risk of bias \\
\hline Mobility & 6 & 514 & $0.25(0.02$ to 0.48$)$ & Moderate; downgraded for risk of bias \\
\hline Health & 3 & 310 & $-0.05(-0.42$ to 0.33$)$ & $\begin{array}{l}\text { Low; downgraded for risk of bias and } \\
\text { imprecision }\end{array}$ \\
\hline \multirow[t]{2}{*}{ Frailty } & 2 & 213 & $-0.41(-0.68$ to -0.14$)$ & Moderate; downgraded for risk of bias \\
\hline & 3 & 359 & RR $0.72(0.52$ to 1.00$)$ 川 & Moderate; downgraded for risk of bias \\
\hline Diet quality & 2 & 141 & $0.53(-0.98$ to 2.04$)$ & $\begin{array}{l}\text { Very low; downgraded for risk of bias, } \\
\text { inconsistency and imprecision }\end{array}$ \\
\hline Quality of life & 3 & 267 & $0.31(-0.05$ to 0.67$)$ & $\begin{array}{l}\text { Low; downgraded for risk of bias and } \\
\text { imprecision }\end{array}$ \\
\hline \multicolumn{5}{|c|}{$\begin{array}{l}\text { Note: } \mathrm{Cl}=\text { confidence interval, } \mathrm{RR}=\text { risk ratio, } \mathrm{SMD}=\text { standardized mean difference. } \\
\text { *Physical function: activities of daily living, muscle strength (handgrip and nonhandgrip) and appendicular lean mass; mobility: gait speed, Timed Up and Go test, } \\
\text { Sit-to-Stand test, balance test and Short Physical Performance Battery; health: body weight and body mass index; diet quality: energy intake in calories. } \\
\text { †For all interventions, there were no data for death, use of health care services, or caregiver and social support. For protein supplementation interventions, there were no } \\
\text { data for quality of life. } \\
\text { †Except where noted otherwise. } \\
\text { §Large: } \sim 0.8 ; \text { medium: } \sim 0.5 ; \text { small: } \sim 0.2 \text {. } \\
\text { qDichotomous outcome. }\end{array}$} \\
\hline
\end{tabular}

\section{Combined-approach interventions}

The nutrition component of combined-approach interventions ${ }^{29-33,35}$ consisted of protein supplementation, provision of food, vitamin D supplementation, dietary counselling, education or cooking classes. The physical activity component was mostly muscle-strengthening exercises through resistance and strength training, but 3 interventions ${ }^{23,30,34}$ also included aerobic exercises (Appendix 4, Supplemental Table S2). Together, the combined-approach interventions had small but significant effects on physical, mobility and frailty measures, with moderate certainty of evidence (Table 2; Appendix 6, Supplemental Table S6). There were no significant effects on measures of health, diet qual- ity or quality of life (Table 2; Appendix 8, Supplemental Figures S13-S15).

The same 6 interventions had data for both physical and mobility meaures. ${ }^{29-33,35}$ These interventions involved 258 intervention and 256 control participants and ranged in duration from 12 to 52 weeks. They had small but significant pooled effect estimates for physical measures (SMD 0.19, $95 \%$ CI 0.06 to 0.32 ) (Figure $4 \mathrm{~A}$ ) and mobility measures (SMD $0.25,95 \%$ CI 0.02 to 0.48 ) (Figure 4B).

Frailty outcomes were reported in 2 RCTs (100 intervention and 113 control participants). ${ }^{30,31}$ There was a small but significant pooled effect estimate for frailty (SMD $-0.41,95 \%$ CI -0.68 to -0.14 ) (Figure 4C). Frailty was also measured as 


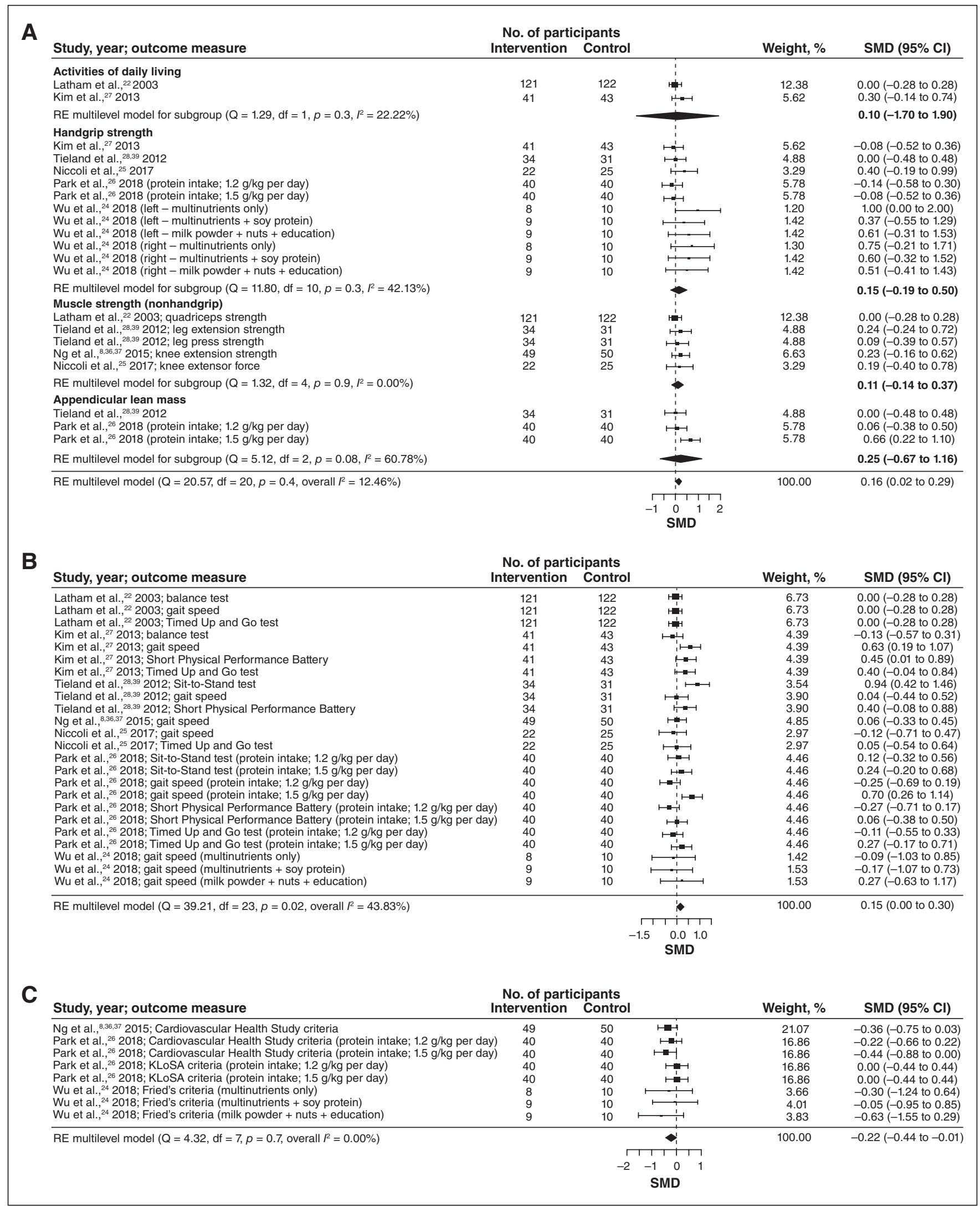

Figure 2: Effects of nutrition interventions on measures of physical outcomes (A), mobility (B) and frailty (C). Weights are from random-effects $(\mathrm{RE})$ multilevel model analysis. Note: $\mathrm{Cl}=$ confidence interval, $\mathrm{df}=$ degrees of freedom, $\mathrm{KLoSA}=\mathrm{Korean}$ Longitudinal Study of $\mathrm{Aging}$, SMD $=$ standardized mean difference. 
A

Study, year; outcome measure

No. of participants

Activities of daily living

Kim et al., ${ }^{27} 2013$

RE multilevel model for subgroup $\left(\mathrm{Q}=0.00, \mathrm{df}=0, p=1.0, I^{2}=0.00 \%\right)$

Handgrip strength

Kim et al., 272013

Tieland et al., ${ }^{28,39} 2012$

Niccoli et al.,25 2017

Park et al.. ${ }^{26} 2018$ (protein intake; $1.2 \mathrm{~g} / \mathrm{kg}$ per day)

Park et al., ${ }^{26} 2018$ (protein intake; $1.5 \mathrm{~g} / \mathrm{kg}$ per day)

Wu et al., ${ }^{24} 2018$ (left - multinutrients + soy protein)

Wu et al. ${ }^{24} 2018$ (left - milk powder + nuts + education)

Wu et al., ${ }^{24} 2018$ (right - milk powder + nuts + education)

$\mathrm{RE}$ multilevel model for subgroup $\left(\mathrm{Q}=6.96 \mathrm{df}=8, p=0.5, l^{2}=28.52 \%\right)$

Muscle strength (nonhandgrip)

Tieland et al., 28,39 2012; leg extension strength

Tieland et al., 28,39 2012; leg press strength

Niccoli et al., ${ }^{25} 2017$; knee extensor force

RE multilevel model for subgroup $\left(\mathrm{Q}=0.19, \mathrm{df}=2, p=0.9, I^{2}=0.00 \%\right)$

Appendicular lean mass

Tieland et al., ${ }^{28,39} 2012$

Park et al. ${ }^{26} 2018$ (protein intake; $1.2 \mathrm{~g} / \mathrm{kg}$ per day)

Park et al., ${ }^{26} 2018$ (protein intake; $1.5 \mathrm{~g} / \mathrm{kg}$ per day)

Intervention Control

Weight, \%

SMD $(95 \% \mathrm{Cl})$

\begin{tabular}{llll} 
SIMD (95\% Cl) \\
\hline$\%)$
\end{tabular}

$\mathrm{RE}$ multilevel model for subgroup $\left(\mathrm{Q}=5.12, \mathrm{df}=2, p=0.08, I^{2}=60.78 \%\right.$ )

RE multilevel model $\left(\mathrm{Q}=13.94, \mathrm{df}=15, p=0.5\right.$, overall $\left.{ }^{2}=6.11 \%\right)$

B

Study, year; outcome measure $\begin{gathered}\text { No. of participants } \\ \text { Intervention Control }\end{gathered}$

\section{Kim et al., ${ }^{27} 2013$; balance test}

Kim et al., ${ }^{27} 2013$; gait speed

Kim et al., ${ }^{27}$ 2013; Short Physical Performance Battery

Kim et al., ${ }^{27}$ 2013; Timed Up and Go test

Tieland et al.,.8,39 2012; Sit-to-Stand test

Tieland et al., ${ }^{28,39}$ 2012; gait speed

Tieland et al., 28,39 2012; Short Physical Performance Battery

Niccoli et al., ${ }^{25} 2017$; gait speed

Niccoli et al., ${ }^{25}$ 2017; Timed Up and Go test

Park et al., ${ }^{26}$ 2018; Sit-to-Stand test (protein intake; $1.2 \mathrm{~g} / \mathrm{kg}$ per day)

Park et al. ${ }^{26}$ 2018: Sit-to-Stand test (protein intake; $15 \mathrm{~g} / \mathrm{kg}$ per day)

Park et al., ${ }^{26} 2018$; gait speed (protein intake; $1.2 \mathrm{~g} / \mathrm{kg}$ per day)

Park et al., ${ }^{26} 2018$; gait speed (protein intake; $1.5 \mathrm{~g} / \mathrm{kg}$ per day)

Park et al., ${ }^{26}$ 2018; Short Physical Performance Battery (protein intake; $1.2 \mathrm{~g} / \mathrm{kg}$ per day)

Park et al., ${ }^{26}$ 2018; Short Physical Performance Battery (protein intake; $1.5 \mathrm{~g} / \mathrm{kg}$ per day)

Park et al. ${ }^{26}$ 2018; Timed Up and Go test (protein intake; $1.2 \mathrm{~g} / \mathrm{kg}$ per day)

Park et al., ${ }^{26} 2018$; Timed Up and Go test (protein intake; $1.5 \mathrm{~g} / \mathrm{kg}$ per day)

Wu et al., ${ }^{24}$ 2018; gait speed (multinutrients + soy protein)

Wu et al., ${ }^{24}$ 2018; gait speed (milk powder + nuts + education)

RE multilevel model $\left(\mathrm{Q}=34.94, \mathrm{df}=18, p=0.01\right.$, overall $\left.I^{2}=49.95 \%\right)$

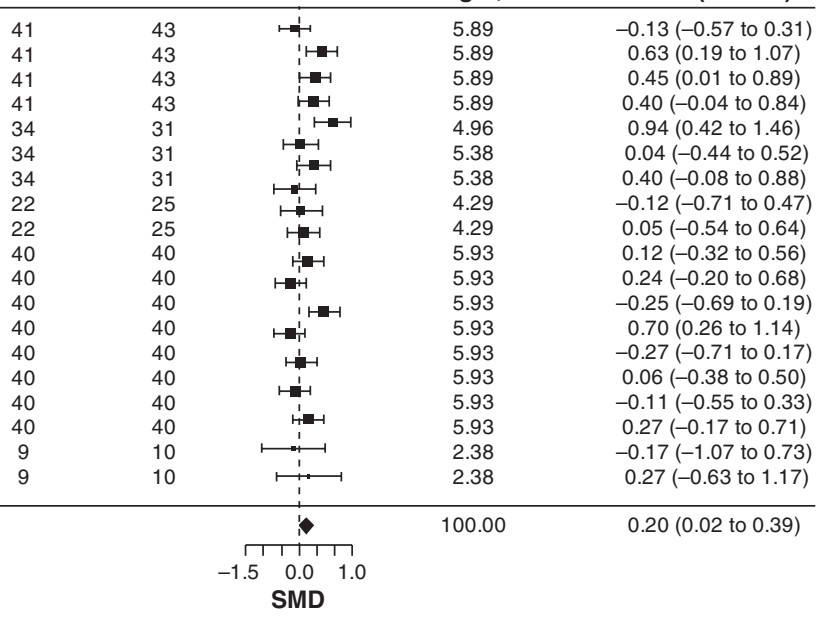

Figure 3: Effects of protein supplementation interventions on measures of physical outcomes (A) and mobility (B). Weights are from randomeffects (RE) multilevel model analysis. Note: $\mathrm{Cl}=$ confidence interval, SMD = standardized mean difference.

prevalence of frailty after the intervention in $3 \mathrm{RCTs}^{30,31,34}$ with 174 intervention and 185 control participants; they ranged in duration from 12 to 52 weeks. The pooled effect estimate showed a significant reduction in this outcome (RR $0.72,95 \%$ CI 0.52 to 1.00 ) (Figure 4D).

\section{Interpretation}

Our review showed a small but significant benefit of nutrition (including protein supplementation) and combined-approach interventions compared to control groups for physical and mobility outcomes, based on moderate certainty of evidence.
Our analysis for nutrition and combined-approach interventions showed a small but significant benefit on frailty outcomes, based on moderate certainty of evidence.

Previous systematic reviews and meta-analyses evaluating the benefit of various interventions and their components in frailty prevention and reversal focused on specific settings ${ }^{10,48}$ or specific interventions, primarily physical activity interventions. ${ }^{9,49}$ Others included a broader mix of interventions, ${ }^{5,6,50}$ which made their assessment more heterogeneous than our review. Some reviews lacked meta-analyse ${ }^{11,51,52}$ or focused on only a few specific outcomes, such as physical performance, ${ }^{11}$ body composition, leg strength and walking speed..$^{53}$ Some 
A

Study, year; outcome measure

No. of participants

Intervention Control

Activities of daily living

Serra-Prat et al., ${ }^{30} 2017$

RE multilevel model for subgroup $\left(\mathrm{Q}=0.00, \mathrm{df}=0, p=1.0, I^{2}=0.00 \%\right)$

Handgrip strength

Tieland et al., ${ }^{28,39} 2012$

Yamada et al. ${ }^{35} 2012$

Kwon et al., ${ }^{32} 2015$

Luger et al., ${ }^{31,41-44} 2016$

Serra-Prat et al., ${ }^{30} 2017$ (men)

Serra-Prat et al., ${ }^{30} 2017$ (women)

Kang et al., ${ }^{29} 2019$ (men)

Kang et al., ${ }^{29} 2019$ (women)

RE multilevel model for subgroup $\left(\mathrm{Q}=3.28, \mathrm{df}=7, p=0.9, I^{2}=0.00 \%\right)$

Muscle strength (nonhandgrip)

Tieland et al., ${ }^{28,39}$ 2012; leg extension strength

Tieland et al., ${ }^{28,39}$ 2012; leg press strength

Yamada et al., ${ }^{35}$ 2012; knee extension torque

Luger et al., ${ }^{31,41-44} 2016$; lower limb strength

RE multilevel model for subgroup $\left(\mathrm{Q}=3.13, \mathrm{df}=3, p=0.4, l^{2}=33.01 \%\right)$

\section{Appendicular lean mass}

Tieland et al. ${ }^{28,39} 2012$

Yamada et al. ${ }^{35} 2012$

Luger et al., ${ }^{31,41-44} 2016$

RE multilevel model for subgroup $\left(\mathrm{Q}=1.85, \mathrm{df}=2, p=0.4, l^{2}=0.31 \%\right)$

RE multilevel model $\left(\mathrm{Q}=9.54, \mathrm{df}=15, p=0.8\right.$, overall $\left.l^{2}=0.00 \%\right)$

\begin{tabular}{|c|c|c|c|c|}
\hline & & $i$ & & \\
\hline \multirow[t]{2}{*}{61} & 72 & $1+$ & 11.98 & $0.04(-0.30$ to 0.38$)$ \\
\hline & & & & $0.04(-0.30$ to 0.38$)$ \\
\hline 31 & 31 & 尚 & 5.13 & $0.24(-0.28$ to 0.76$)$ \\
\hline 35 & 35 & $1+1$ & 5.99 & $0.19(-0.29$ to 0.67$)$ \\
\hline 26 & 28 & 1.- & 5.13 & $0.27(-0.25$ to 0.79$)$ \\
\hline 39 & 41 & $t=1$ & 7.19 & $0.37(-0.07$ to 0.81$)$ \\
\hline 30 & 28 & $\frac{1}{1}-1$ & 5.13 & $0.31(-0.21$ to 0.83$)$ \\
\hline 31 & 44 & $\mapsto-1$ & 5.99 & $-0.15(-0.63$ to 0.33$)$ \\
\hline 25 & 19 & $\stackrel{1}{\mapsto-1}$ & 3.99 & $0.21(-0.38$ to 0.80$)$ \\
\hline \multirow[t]{2}{*}{41} & 30 & $\mapsto$ & 5.99 & $0.04(-0.44$ to 0.52$)$ \\
\hline & & $\omega$ & & $0.18(-0.03$ to 0.40$)$ \\
\hline 31 & 31 & $\stackrel{1}{\mapsto-1}$ & 5.99 & -0.01 ( -0.49 to 0.47$)$ \\
\hline 31 & 31 & $\mapsto$ & 5.99 & $-0.03(-0.51$ to 0.45$)$ \\
\hline 35 & 35 & फ्- & 5.99 & $0.30(-0.18$ to 0.78$)$ \\
\hline \multirow[t]{2}{*}{39} & 41 & $1=-1$ & 7.19 & 0.46 (0.02 to 0.90$)$ \\
\hline & & & & $0.22(-0.28$ to 0.71$)$ \\
\hline 31 & 31 & $i_{1}=1$ & 5.13 & $0.31(-0.21$ to 0.83$)$ \\
\hline 35 & 35 & in- & 5.99 & $0.53(0.05$ to 1.01$)$ \\
\hline \multirow[t]{3}{*}{39} & 41 & $\mapsto-1$ & 7.19 & $0.08(-0.36$ to 0.52$)$ \\
\hline & & & & $0.29(-0.31$ to 0.90$)$ \\
\hline & & 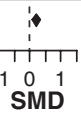 & 100.00 & 0.19 ( 0.06 to 0.32$)$ \\
\hline
\end{tabular}

B

Study, year; outcome measure

No. of participants

Tieland et al., ${ }^{28,39}$ 2012; Sit-to-Stand tes

Tieland et al., ${ }^{28,39}$ 2012; gait speed

Tieland et al., 28,39 2012; Short Physical Performance Battery

Yamada et al., ${ }^{35}$ 2012; Sit-to-Stand test

Yamada et al., ${ }^{35}$ 2012; gait speed

Yamada et al., 35 2012; Timed Up and Go test

Kwon et al., ${ }^{32}$ 2015; gait speed

Luger et al., ${ }^{31,41-44}$ 2016; balance test

Luger et al., ${ }^{31,41-44}$ 2016; gait speed

Luger et al., ${ }^{31,41-44}$ 2016; Short Physical Performance Battery

Serra-Prat et al., ${ }^{30} 2017$; gait speed

Serra-Prat et al., ${ }^{30}$ 2017; Timed Up and Go test

Kang et al. ${ }^{29} 2019$; Sit-to-Stand test

Kang et al., ${ }^{29} 2019$; gait speed

RE multilevel model $\left(\mathrm{Q}=28.76, \mathrm{df}=13, p=0.007\right.$, overall $\left.l^{2}=56.89 \%\right)$

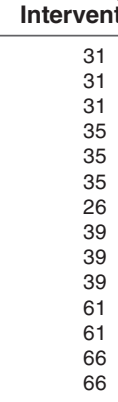

31
31
31
35
35
35
26
39
39
39
61
61
66
66

31
31
31
35
35
35
28
41
41
41
72
72
49
49

C

Study, year; outcome measure

No. of participants

Luger et al 31,41-44 2016; Fried's criteria (SHARE-FI)

Serra-Prat et al., ${ }^{30}$ 2017; modified Fried's criteria

Intervention Control

$\operatorname{SMD}(95 \% \mathrm{Cl})$

$-0.37(-0.89$ to 0.15$)$

$0.00(-0.48$ to 0.48$)$

$0.11(-0.37$ to 0.59$)$

$0.09(-0.39$ to 0.57$)$

$0.28(-0.20$ to 0.76$)$

$0.37(-0.11$ to 0.85$)$

$0.11(-0.41$ to 0.63$)$

$-0.09(-0.53$ to 0.35$)$

$0.27(-0.17$ to 0.71$)$

$0.31(-0.13$ to 0.75$)$

$0.70(0.36$ to 1.04$)$

$0.00(-0.34$ to 0.34$)$

$0.81(0.42$ to 1.20$)$

$0.53(0.14$ to 0.92$)$

$\begin{array}{ll}7.81 & 0.53(0.14 \text { to } 0.92) \\ 100.00 & 0.25(0.02 \text { to } 0.48)\end{array}$

DL RE model $\left(\mathrm{Q}=0.32, \mathrm{df}=1, p=0.6\right.$, overall $\left.l^{2}=0.00 \%\right)$

$\begin{array}{ll}39 & 41 \\ 61 & 72\end{array}$

\begin{tabular}{rrrr}
41 & 37.50 & $-0.31(-0.75$ to 0.13$)$ \\
72 & 62.50 & $-0.47(-0.81$ to -0.13$)$ \\
\hline & 100.00 & $-0.41(-0.68$ to -0.14$)$ \\
\hline & & &
\end{tabular}

SMD

\section{Study, year; outcome measure}

Intervention,

no. of participants no. of participants

Nykänen et al. ${ }^{34}$ 2012; Cardiovascular Health Study criteria

Luger et al. 31,41-44 2016. Fried's criteria

Serra-Prat et al. ${ }^{30} 2017$; Fried's criteria

Frail Total

Frail Total

Weight, \%

RR (95\% Cl)

$\begin{array}{cccc}\text { Frail } & \text { Total } & \text { Frail } & \text { To } \\ 19 & 74 & 27 & 72\end{array}$

DL RE model $\left(\mathrm{Q}=2.18, \mathrm{df}=2, p=0.3\right.$, overall $\left.l^{2}=0.00 \%\right)$

$\begin{array}{cccc}19 & 74 & 27 & 72 \\ 17 & 39 & 21 & 41 \\ 3 & 61 & 11 & 72\end{array}$

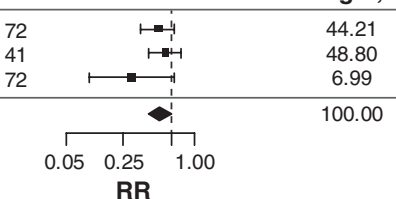

RR

Figure 4: Effects of combined nutrition and physical activity interventions on measures of physical outcomes (A), mobility (B) and frailty

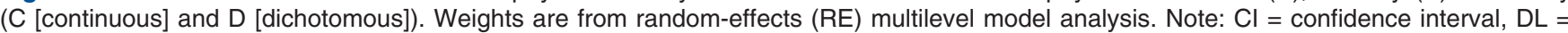
DerSimonian and Laird, RR = risk ratio, SHARE-FI = Frailty Instrument of the Survey of Health, Ageing and Retirement in Europe, SMD = standardized mean difference. 
reviews of nutrition interventions included participants who were malnourished or sarcopenic and not necessarily frail. ${ }^{54-56}$ To address these gaps in the literature, we focused on nutrition and combined-approach studies with participants clearly identified as prefrail or frail, and looked at the benefit of these interventions for a more robust collection of outcomes.

We found mixed effects depending on intervention type and outcomes. Protein supplementation in older adults is a priority, given that many consume less than the recommended daily amounts. ${ }^{25}$ Like other investigators, ${ }^{1,50}$ we found that protein supplementation RCTs had significant effects on physical and mobility measures, but not frailty. It has been suggested that, for nutrition interventions to be beneficial on their own, without the addition of physical activity, the population may need to be at deficiency or malnourished ${ }^{1,57}$ and at similar levels of baseline frailty; ${ }^{1}$ however, there is a lack of evidence for this second hypothesis.

The importance of protein supplementation in combination with resistance exercise in healthy older adults has also been established by evidence and reviews. Our review is in agreement with those showing that nutrition interventions combined with physical activity are effective at decreasing frailty and improving gait speed, grip strength and physical performance. ${ }^{48,50}$ Although our effect estimates for these interventions were slightly higher than those for nutrition alone, we did not see any additional outcomes of significance with these intervention types, and, overall, the effect estimates were small. Therefore, our analysis does not show that exercise adds significantly to nutrition interventions.

Although our review was selective in defining the population of interest, research is still lacking in the explicit identification of prefrail and frail older adults, and consistency in how these tools are applied. Very few researchers in our review measured frailty as a postintervention outcome. Likewise, the wide range of outcome measures that were used across studies may reflect the lack of clarity in frailty identification and diagnosis. Owing to this lack of clarity, we could not evaluate the effectiveness of interventions by level of frailty, which affected our interpretation of the clinical effectiveness of the studies. However, our use of SMDs provides a magnitude of the effect, which has implications for clinical practice. Welldesigned clinical trials that have clear definitions of frailty and use outcomes reflecting frailty identification and diagnosis are needed to inform interventions that prevent or delay frailty progression in older adults.

\section{Limitations}

Although our search was comprehensive, we did not explicitly search the literature for combined interventions that included nutrition with physical activity components, so we may have missed potentially relevant studies. We used the strict criterion of frailty in an attempt to identify a more homogeneous population, but it led to a large number of exclusions, which may have included potentially frail populations or those with overlapping conditions. We extracted outcomes immediately after interventions, which leaves the long-lasting effects of these interventions unknown. The protocol of our review has limitations, including restriction to English-language citations, our search end date of July 2019 and the fact that we did not search the grey literature. Last, although we did not observe any significant asymmetry across funnel plots for publication bias, studies were small (<250 participants) and had risk of bias concerns. The small samples also meant that prefrail and frail participants were grouped together, which did not allow for subanalysis on frailty level.

Although there may be concerns about the reuse of the same participants from the same study to contribute data for multiple measures of a given outcome, our group agreed that this approach is preferred over selective reporting of effect sizes for a given outcome or averaging outcome measures from the same study to conduct a conventional 2-level metaanalysis. Both selective reporting (i.e., choosing 1 outcome measure for a given outcome) and averaging all outcome measures result in the potential loss and dilution of relevant information, and may produce misleading, inaccurate and biased results. These ad hoc approaches may also lead to missed opportunities to use all available data to address the relevant research questions. ${ }^{58}$

\section{Conclusion}

This review adds to the body of evidence identifying interventions that benefit components of frailty, such as physical and mobility outcomes, and frailty itself. However, our use of a strict inclusion criterion that identified a prefrail or frail population shows that future studies need to better define frailty, to ensure improved identification of older adults who would benefit from such interventions. This would facilitate the adoption and implementation of effective interventions into clinical practice.

\section{References}

1. Dent E, Morley JE, Cruz-Jentoft AJ, et al. Physical frailty: ICFSR international clinical practice guidelines for identification and management. 7 Nutr Health Aging 2019;23:771-87.

2. Muscedere J, Andrew MK, Bagshaw SM, et al. Screening for frailty in Canada's health care system: a time for action. Can F Aging 2016;35:281-97.

3. Kehler DS, Ferguson T, Stammers AN, et al. Prevalence of frailty in Canadians 18-79 years old in the Canadian Health Measures Survey. BMC Geriatr 2017;17:28.

4. Morley JE, Vellas B, van Kan GA, et al. Frailty consensus: a call to action. $\mathcal{f}$ Am Med Dir Assoc 2013;14:392-7.

5. Puts MTE, Toubasi S, Andrew MK, et al. Interventions to prevent or reduce the level of frailty in community-dwelling older adults: a scoping review of the literature and international policies. Age Ageing 2017;46:383-92.

6. Negm AM, Kennedy CC, Thabane L, et al. Management of frailty: a systematic review and network meta-analysis of randomized controlled trials. $7 \mathrm{Am}$ Med Dir Assoc 2019;20:1190-8.

7. Clegg A, Young J, Iliffe S, et al. Frailty in elderly people. Lancet 2013;381:752-62.

8. Ng TP, Feng L, Nyunt MSZ, et al. Nutritional, physical, cognitive, and combination interventions and frailty reversal among older adults: a randomized controlled trial. Am 7 Med 2015;128:1225-36.e1.

9. Jadczak AD, Makwana N, Luscombe-Marsh N, et al. Effectiveness of exercise interventions on physical function in community-dwelling frail older people: an umbrella review of systematic reviews. $7 B I$ Database System Rev Implement Rep 2018;16:752-75.

10. Macdonald SH, Travers J, Shé ÉN, et al. Primary care interventions to address physical frailty among community-dwelling adults aged 60 years or older: a meta-analysis. PLoS One 2020;15:e0228821.

11. Kidd T, Mold F, Jones C, et al. What are the most effective interventions to improve physical performance in pre-frail and frail adults? A systematic review of randomised control trials. BMC Geriatr 2019;19:184.

12. Racey MA, Ali MU, Sherifali D, et al.; Canadian Frailty Network. Effectiveness of physical activity interventions in older adults with frailty or prefrailty: a systematic review and meta-analysis. CMA7 Open 2021;9:E728-43. 
13. Moher D, Liberati A, Tetzlaff J, et al. Preferred reporting items for systematic reviews and meta-analyses: the PRISMA statement. 7 Clin Epidemiol 2009;62: 1006-12.

14. Kim H, Awata S, Watanabe Y, et al. Cognitive frailty in community-dwelling older Japanese people: prevalence and its association with falls. Geriatr Gerontol Int 2019;19:647-53.

15. Higgins JPT, Altman DG, Gøtzsche PC, et al. The Cochrane Collaboration's tool for assessing risk of bias in randomised trials. BM7 2011;343:d5928.

16. Schünemann $\mathrm{H}$, Bro zek J, Guyatt $\mathrm{G}$, et al., editors. GRADE handbook for grading quality of evidence and strength of recommendations. London (UK): GRADE Working Group; updated 2013.

17. DerSimonian R, Laird N. Meta-analysis in clinical trials. Control Clin Trials 1986; 7:177-88.

18. Higgins JPT, Li T, Deeks JJ. Choosing effect measures and computing estimates of effect. In: Higgins JPT, Thomas J, Chandler J, et al., editors. Cochrane bandbook for systematic reviews of interventions version 6.1. London (UK): Cochrane; updated 2021:143-76.

19. Assink M, Wibbelink CJM. Fitting three-level meta-analytic models in R: a step-by-step tutorial. Quant Methods Psychol 2016;12:154-74.

20. Cohen J. Statistical power analysis for the bebavioral sciences. 2nd ed. Mahwah (NJ): Lawrence Erlbaum Associates; 1988.

21. Pastor DA, Lazowski RA. On the multilevel nature of meta-analysis: a tutorial, comparison of software programs, and discussion of analytic choices. Multivariate Behav Res 2018;53:74-89.

22. Latham NK, Anderson CS, Lee A, et al.; Fitness Collaborative Group. A randomized, controlled trial of quadriceps resistance exercise and vitamin D in frail older people: the Frailty Interventions Trial in Elderly Subjects (FITNESS). 7 Am Geriatr Soc 2003;51:291-9.

23. de Jong N, Paw MJCA, de Graaf C, et al. Effect of dietary supplements and physical exercise on sensory perception, appetite, dietary intake and body weight in frail elderly subjects. Br 7 Nutr 2000;83:605-13.

24. Wu SY, Hsu LL, Hsu CC, et al. Dietary education with customised dishware and food supplements can reduce frailty and improve mental well-being in elderly people: a single-blind randomized controlled study. Asia Pac 7 Clin Nutr 2018;27:1018-30.

25. Niccoli S, Kolobov A, Bon T, et al. Whey protein supplementation improves rehabilitation outcomes in hospitalized geriatric patients: a double blinded, randomized controlled trial. 7 Nutr Gerontol Geriatr 2017;36:149-65.

26. Park Y, Choi JE, Hwang HS. Protein supplementation improves muscle mass and physical performance in undernourished prefrail and frail elderly subjects: a randomized, double-blind, placebo-controlled trial. Am 7 Clin Nutr 2018; 108:1026-33

27. Kim CO, Lee KR. Preventive effect of protein-energy supplementation on the functional decline of frail older adults with low socioeconomic status: a community-based randomized controlled study. 7 Gerontol A Biol Sci Med Sci 2013;68:309-16.

28. Tieland M, van de Rest O, Dirks ML, et al. Protein supplementation improves physical performance in frail elderly people: a randomized, double-blind, placebo-controlled trial. 7 Am Med Dir Assoc 2012;13:720-6.

29. Kang L, Gao Y, Liu X, et al. Effects of whey protein nutritional supplement on muscle function among community-dwelling frail older people: a multicenter study in China. Arch Gerontol Geriatr 2019;83:7-12.

30. Serra-Prat M, Sist X, Domenich R, et al. Effectiveness of an intervention to prevent frailty in pre-frail community-dwelling older people consulting in primary care: a randomised controlled trial. Age Ageing 2017;46:401-7.

31. Luger E, Dorner TE, Haider S, et al. Effects of a home-based and volunteeradministered physical training, nutritional, and social support program on malnutrition and frailty in older persons: a randomized controlled trial. $7 \mathrm{Am} \mathrm{Med}$ Dir Assoc 2016;17:671.e9-16.

32. Kwon J, Yoshida Y, Yoshida H, et al. Effects of a combined physical training and nutrition intervention on physical performance and health-related quality of life in prefrail older women living in the community: a randomized controlled trial. 7 Am Med Dir Assoc 2015;16:263.e1-8.

33. Tieland M, Dirks ML, van der Zwaluw N, et al. Protein supplementation increases muscle mass gain during prolonged resistance-type exercise training in frail elderly people: a randomized, double-blind, placebo-controlled trial. $\mathfrak{f}$ Am Med Dir Assoc 2012;13:713-9.

34. Nykänen I, Rissanen TH, Sulkava R, et al. Effects of individual dietary counseling as part of a comprehensive geriatric assessment (CGA) on frailty status: a population-based intervention study. 7 Clin Gerontol Geriatr 2012;3:89-93.

35. Yamada M, Arai H, Yoshimura K, et al. Nutritional supplementation during resistance training improved skeletal muscle mass in community-dwelling frail older adults. F Frailty Aging 2012;1:64-70.

36. Ng TP, Ling LHA, Feng L, et al. Cognitive effects of multi-domain interventions among pre-frail and frail community-living older persons: randomized controlled trial. 7 Gerontol A Biol Sci Med Sci 2018;73:806-12.

37. Ng TP, Nyunt MSZ, Feng L, et al. Multi-domains lifestyle interventions reduces [sic] depressive symptoms among frail and pre-frail older persons: randomized controlled trial. 7 Nutr Health Aging 2017;21:918-26.

38. van der Zwaluw NL, van de Rest O, Tieland $M$, et al. The impact of protein supplementation on cognitive performance in frail elderly. Eur $\mathcal{F}$ Nutr 2014;53: 803-12.
39. Paw MJCA, de Jong N, Schouten EG, et al. Physical exercise and/or enriched foods for functional improvement in frail, independently living elderly: a randomized controlled trial. Arch Phys Med Rehabil 2001;82:811-7.

40. Paw MJMCA, de Jong N, Schouten EG, et al. Physical exercise or micronutrient supplementation for the wellbeing of the frail elderly? A randomised controlled trial. Br 7 Sports Med 2002;36:126-31.

41. Kapan A, Luger E, Haider S, et al. Fear of falling reduced by a lay led homebased program in frail community-dwelling older adults: a randomised controlled trial. Arch Gerontol Geriatr 2017;68:25-32.

42. Haider S, Grabovac I, Winzer E, et al. Change in inflammatory parameters in prefrail and frail persons obtaining physical training and nutritional support provided by lay volunteers: a randomized controlled trial. PLoS One 2017;12: e0185879.

43. Haider S, Dorner TE, Luger E, et al. Impact of a home-based physical and nutritional intervention program conducted by lay-volunteers on handgrip strength in prefrail and frail older adults: a randomized control trial. PLoS One 2017;12:e0169613.

44. Kapan A, Winzer E, Haider S, et al. Impact of a lay-led home-based intervention programme on quality of life in community-dwelling pre-frail and frail older adults: a randomized controlled trial. BMC Geriatr 2017;17:154.

45. Hangelbroek RWJ, Knuiman P, Tieland M, et al. Attenuated strength gains during prolonged resistance exercise training in older adults with high inflammatory status. Exp Gerontol 2018;106:154-8.

46. Dirks ML, Tieland M, Verdijk LB, et al. Protein supplementation augments muscle fiber hypertrophy but does not modulate satellite cell content during prolonged resistance-type exercise training in frail elderly. 7 Am Med Dir Assoc 2017;18:608-15.

47. Fried LP, Tangen CM, Walston J, et al.; Cardiovascular Health Study Collaborative Research Group. Frailty in older adults: evidence for a phenotype. $f$ Gerontol A Biol Sci Med Sci 2001;56:M146-56.

48. Frost R, Belk C, Jovicic A, et al. Health promotion interventions for community-dwelling older people with mild or pre-frailty: a systematic review and meta-analysis. BMC Geriatr 2017;17:157.

49. Bray NW, Smart RR, Jakobi JM, et al. Exercise prescription to reverse frailty. Appl Physiol Nutr Metab 2016;41:1112-6.

50. Apóstolo J, Cooke R, Bobrowicz-Campos E, et al. Effectiveness of interventions to prevent pre-frailty and frailty progression in older adults: a systematic review. FBI Database System Rev Implement Rep 2018;16:140-232.

51. Manal B, Suzana S, Singh DKA. Nutrition and frailty: a review of clinical intervention studies. F Frailty Aging 2015;4:100-6.

52. Artaza-Artabe I, Saez-Lopez P, Sanchez-Hernandez N, et al. The relationship between nutrition and frailty: effects of protein intake, nutritional supplementation, vitamin D and exercise on muscle metabolism in the elderly. A systematic review. Maturitas 2016;93:89-99.

53. Farooqi V, van den Berg MEL, Cameron ID, et al. Anabolic steroids for rehabilitation after hip fracture in older people. Cochrane Database Syst Rev 2014; (10):CD008887.

54. Veronese N, Stubbs B, Punzi L, et al. Effect of nutritional supplementations on physical performance and muscle strength parameters in older people: a systematic review and meta-analysis. Ageing Res Rev 2019;51:48-54.

55. Lozano-Montoya I, Correa-Pérez A, Abraha I, et al. Nonpharmacological interventions to treat physical frailty and sarcopenia in older patients: a systematic overview: the SENATOR Project ONTOP Series. Clin Interv Aging 2017; 12:721-40.

56. Gomes F, Baumgartner A, Bounoure L, et al. Association of nutritional support with clinical outcomes among medical inpatients who are malnourished or at nutritional risk: an updated systematic review and meta-analysis. $7 A M A$ Netw Open 2019;2:e1915138.

57. Marcucci M, Damanti S, Germini F, et al. Interventions to prevent, delay or reverse frailty in older people: a journey towards clinical guidelines. $\mathrm{BMC} \mathrm{Med}$ 2019;17:193.

58. Cheung MWL. A guide to conducting a meta-analysis with non-independent effect sizes. Neuropsychol Rev 2019;29:387-96.

Affiliations: McMaster Evidence Review and Synthesis Team (Racey, Ali, Sherifali, Fitzpatrick-Lewis, Lewis, Jovkovic); School of Nursing (Racey, Sherifali, Fitzpatrick-Lewis), Department of Clinical Epidemiology \& Biostatistics (Ali) and School of Rehabilitation Science (Tang), Faculty of Health Sciences, McMaster University, Hamilton, Ont.; University of Alberta (Gramlich); Nutrition Services (Gramlich), Alberta Health Services, Edmonton, Alta.; Schlegel-University of Waterloo Research Institute for Aging (Keller); Department of Kinesiology (Keller), University of Waterloo, Waterloo, Ont.; Cumming School of Medicine (Holroyd-Leduc), University of Calgary, Calgary, Alta.; Department of Family and Emergency Medicine (Giguère), Laval University, Québec, Que.; Faculty of Kinesiology (Bouchard), University of New Brunswick; Cardiometabolic Exercise \& Lifestyle Laboratory (Bouchard), Fredericton, NB; Canadian Frailty Network (Prorok, Kim, Lorbergs), Kingston, Ont. 
Contributors: Megan Racey, Muhammad Usman Ali, Diana Sherifali, Donna Fitzpatrick-Lewis, Leah Gramlich, Heather Keller, Jayna Holroyd-Leduc, Anik Giguère, Ada Tang, Danielle Bouchard, Jeanette Prorok, Perry Kim, Amanda Lorbergs and John Muscedere designed the study. Megan Racey, Muhammad Usman Ali, Diana Sherifali, Donna Fitzpatrick-Lewis, Ruth Lewis and Milos Jovkovic acquired and analyzed the data and drafted the manuscript. Megan Racey, Muhammad Usman Ali, Diana Sherifali and Donna Fitzpatrick-Lewis interpreted the data. Leah Gramlich, Heather Keller, Jayna Holroyd-Leduc, Anik Giguère, Ada Tang, Danielle Bouchard, Jeanette Prorok, Perry Kim, Amanda Lorbergs and John Muscedere revised the manuscript critically for important intellectual content. All of the authors approved the final version to be published and agreed to be accountable for all aspects of the work.

Members of the Canadian Frailty Network: John Muscedere (Scientific Director), Carol Barrie (Executive Director), Perry Kim (Assistant Scientific Director), Amanda Lorbergs (Manager, Research and Knowledge Translation), Jeanette Prorok (Manager, Special Projects and Initiatives), Kyle Plumb (Project Manager), Mat LaBranche (Finance \& Administration), Amy Doyle (Manager, Strategic Partnership Development and Communications), Kelsey MacIntosh (Health Promotion Coordinator)

Funding: This research was funded by the McMaster Institute for Research and Aging, the McMaster Evidence Review and Synthesis Team (MERST) and the Canadian Frailty Network (CFN) (Technology Evaluation in the Elderly Network), which is supported by the Government of Canada through the Networks of Centres of Excellence program. The CFN was responsible for the conception of the research question and supported the editing of the manuscript. MERST was independently responsible for data extraction, analysis, interpretation and reporting. Together, these funds supported the work of Megan Racey as a postdoctoral fellow. Diana Sherifali holds the Heather M. Arthur Population Health Research Institute/Hamilton Health Sciences Chair in Interprofessional Health Research, which supported her role in this work.

Disclaimer: No one from the Canadian Frailty Network had any involvement in the data extraction or analysis for the review. All screening of articles, data extraction, and data analysis and interpretation were conducted by the first 6 authors, independent from the steering committee and funders.

Content licence: This is an Open Access article distributed in accordance with the terms of the Creative Commons Attribution (CC BY-NCND 4.0) licence, which permits use, distribution and reproduction in any medium, provided that the original publication is properly cited, the use is noncommercial (i.e., research or educational use), and no modifications or adaptations are made. See: https://creativecommons.org/licenses/by -nc-nd/4.0/.

Data sharing: Data used in this review can be requested for confidential research purposes by contacting the corresponding author(s).

Acknowledgement: The authors thank Angela Eady for developing the search strategy.

Supplemental information: For reviewer comments and the original submission of this manuscript, please see www.cmajopen.ca/content/9/3/ E744/suppl/DC1. 